Communications in Physics, Vol.20, No. 4 (2010), pp. 301-307

\title{
PIEZOELECTRIC EFFECT ON THE ELECTRON MOBILITY IN AN UNINTENTIONALLY DOPED GaN/AIGaN SURFACE QUANTUM WELL
}

\author{
NGUYEN VIET MINH \\ Computational Physics Department, Institute of Engineering Physics, \\ Hanoi University of Technology
}

\begin{abstract}
We present a theoretical study the two-dimensional electron gas (2DEG) at low temperature in an unintentionally doped GaN/AlGaN surface quantum well, taking adequate account of the roughness-induced scattering mechansms and effect due to sheet polarization charges. Within model of surface quantum wells describes by an extended Fang-Howard wave function, we are able to derive an analytic expression for the self-consistent Hartree potential. Thus, we obtained simple expresion describing the enhancement of the 2DEG screening and unscreened potentials for different scattering sources. We studied the piezoelectric effect on the electron mobility in an unintentionally doped (UID) GaN/AlGaN surface quantum well.
\end{abstract}

\section{INTRODUCTION}

A surface quantum well GaN/AlGaN is formed by two potentional barriers, the first one is vacuum, and the second one is in the interface between well - GaN and barrierAlGaN. Recently experimental reports indicated that a 2DEG is formed at the naked surface of several semiconductors, such as $\mathrm{ZnO}$ [1-4] InP [5], InGaAs [6, 7], SiGe [8] and GaN [9]. This open structure is referred to as a surface quantum well (SFQW) [10], in which a very high potential barrier $(\sim 4.5 \mathrm{eV})$ between the vacuum and the host crystal leads to an enhanced carrier confinement, i.e., a strong lateral quantization.

An understanding of SFQW is obviously important also for the modeling of lateral quantization in other open system, e.g, quantum wires and quantum dots. However, it should be mentioned that SFQW have been much less studied than quantum wells (QWs).

Thus, this paper is devoted to the development of a theory for piezoelectric effect on the mobility of the two-dimensional electron gas 2DEG at low temperature in an unintentionally doped GaN/AlGaN surface quantum well.

\section{THE ELECTRON MOBILITY DUE TO PIEZOELECTRIC SCATTERING IN UID GaN/AIGaN SFQW}

In what follow, we will be dealing with a UID GaN/AlGaN SFQW. The crystal reference system is that the $\mathrm{z}$ axis is directed from vacuum to the well, and $z=0$ defines the plane between the vacuum $(z<0)$ and the GaN well $(z>0)$. It is assumed that the GaN layer is under tensile strain, while the AlGaN layer is relaxed. The electrons are confined in a QW separated from the vacuum by two potential barriers: one at $z<0$ and 
another at $z=L$, with of well. The barrier height between the vacuum and GaN is very large $\left(V_{0} \sim 5 \mathrm{eV}\right)[9]$, so that the penetration of electrons into the vacuum is negligible. Therefore, the 2DEG in the lowest subband of a GaN/AlGaN SFQW is described by a modified Fang-Howard wave function, proposed by Ando [11,12]:

$$
\zeta(z)=\left\{\begin{array}{lc}
0 & \text { if } \quad z<0 \\
B k^{3 / 2} z e^{-k z / 2} & 0<z<L \\
A \kappa^{1 / 2} e^{-\kappa(z-L)} & z>L
\end{array}\right.
$$

in which $A, B, k, \kappa$ are variational parameters to be determined. Here $\mathrm{k}$ and $\kappa$ are haft the wave numbers in the well and barrier, respectively. $A, B$ are dimensionless parameters. Variational parameters $A, B, \kappa$ and the wave function (1) are determined by $k$ through boundary conditions at $z=L$, and the normalization. And then, $k$ is determined by condition, in which the wave function (1) is to minimize the total energy per particle, which is determined by the Hamiltonian:

$$
H=T+V_{\text {tot }}(z)
$$

where $T$ is the kinetic energy, and $V_{\text {tot }}(z)$ is the total effective confining potential [13]:

$$
V_{\mathrm{tot}}(z)=V_{\mathrm{w}}(z)+V_{b}(z)+V_{H}(z)+V_{i m}(z)+V_{\sigma}(z)+V_{\mathrm{xc}}(z),
$$

in which $V_{\mathrm{w}}(\mathrm{z})$ and $V_{b}(\mathrm{z})$ are the barrier potentials at $z=0$, and $z=L$, the third term $V_{H}(\mathrm{z})$ is the Hartree potential due to ionized donors and confined electrons themselves. The fourth term $V_{i m}(z)$ in Eq. (3) is the potential due to image charge, which quantities the effect arising from an abrupt decrease in the dielectric constant across the surface $z=0$. The fifth term $V_{\sigma}(z)$ is potential of polarization of charges located in an extremely narrow region of the AlGaN barrier near to the interface plane between the GaN well and the AlGaN barrier and their density is positive for all values of $\mathrm{Al}$ content. At last, the exchange-correlation correction $V_{\mathrm{xc}}(z)$ allows the many-body effect in the 2DEG along the normal direction $[12,14]$.

\section{II.1. The electron mobility at low temperature}

The electron mobility at very low temperature may be determined within the relaxation time approximation by

$$
\mu=e \tau / m^{*}
$$

with $m^{*}$ as the in-plane effective electron mass of the GaN [15]. The inverse relaxation time for zero temperature is then expressed in terms of the autocorrelation function for each disorder [12]:

$$
\frac{1}{\tau}=\frac{1}{2 \pi \hbar E_{F}} \int_{0}^{2 k_{F}} d q \frac{q^{2}}{\left(4 k_{F}^{2}-q^{2}\right)^{1 / 2}} \frac{\left\langle|U(\mathbf{q})|^{2}\right\rangle}{\varepsilon^{2}(q)},
$$

where $q=2 k_{F} \sin (\theta / 2)$ as the $2 \mathrm{D}$ momentum transfer by a scattering event in the $x-y$ plane, with $\theta$ as a scattering angle. The Fermi energy is given by $E_{F}=\hbar^{2} k_{F}^{2} / 2 m^{*}$, with $k_{F}$ as the Fermi wave number fixed by the 2DEG density: $k_{F}=\sqrt{2 \pi n_{s}} \cdot\left\langle|U(\mathbf{q})|^{2}\right\rangle$ is autocorrelation function in wave vector space, that is specified for the different random scattering fields. Hereafter, the angular brackets stand for an ensemble average. $U(\mathbf{q})$ is a $2 \mathrm{D}$ Fourier transform of the unscreened scattering potential averaged with the envelope 
wave function of a $2 \mathrm{D}$ subband. The dielectric function $\varepsilon(q)$ entering in Eq. (5) takes account of the screening of a scattering potential by the 2DEG. As usual, this is evaluated within the random phase approximation [14]

$$
\varepsilon(q)=1+\frac{q_{s}}{q} F_{S}(q)[1-G(q)], \quad \text { for } q \leq 2 k_{F} .
$$

Here the inverse 2D Thomas-Fermi screening length is

$$
q_{s}=\frac{2 m^{*} e^{2}}{\varepsilon_{L} \varepsilon_{+} \hbar^{2}}
$$

where by definition

$$
\varepsilon_{ \pm}=\frac{\varepsilon_{L} \pm 1}{\varepsilon_{L}}, \quad \varepsilon_{+}+\varepsilon_{-}=1 .
$$

We introduced the dimensionless wave numbers:

$$
t=q L, \quad a=k L, \quad \text { and } \quad b=\kappa L .
$$

The screening form factor $F_{S}(q)$ takes account of the extension of electronic states along the normal direction. With the wave function (1), we obtained

$$
\begin{aligned}
F_{S}(t)= & \varepsilon\left\{\left[\frac { 1 } { 2 } \left(\frac { 1 } { 2 L } \left(\frac{A^{4} b L(3 b-t)}{b^{2}-t^{2}}+\frac{a B^{4} e^{-2(a+t)} L\left(e^{2 a+t}(a-t)^{3}\left(8 a^{2}+9 a t+3 t^{2}\right)\right.}{\left(a^{2}-t^{2}\right)^{3}}\right.\right.\right.\right. \\
& -8 a^{5} e^{a}\left(2+a^{2}+2 t+t^{2}+2 a(1+t)\right)+e^{t}(a+t)^{3}\left(2 a^{6}-4 a^{5}(t-2)+3 t^{2}\right. \\
& \left.\left.\left.+3 a t(2 t-3)+2 a^{4}\left(8-6 t+t^{2}\right)+2 a^{3}\left(8-9 t+2 t^{2}\right)+2 a^{2}\left(4-9 t+3 t^{2}\right)\right)\right)\right) \\
& +a^{3} A^{2} b B^{2} e^{-a}\left\{\frac{2+a^{2}+2 b+b^{2}+2 a(1+b)-2 e^{a+b}}{(a+b)^{3}(b-t)}\right. \\
& +\frac{2+a^{2}-2 e^{a+t}+2 t+t^{2}+2 a(1+t)}{(a+t)^{3}(t-b)} \\
& +\frac{e^{-t}\left(-2 e^{a}+e^{t}\left(2+a^{2}-2 a(t-1)-2 t+t^{2}\right)\right)}{(a+b)^{3}(b-t)} \\
& -\left(\frac{1}{(a+b)^{3}(a-t)^{3}\left(t^{2}-b^{2}\right)}\right)\left[e ^ { - t } \left(2(a+b)^{3} e^{a}(b-t)+e^{t}(b+t)\left(4 t^{3}\right.\right.\right. \\
& +4 b(-1+t) t^{2}-a^{5}+a^{4}(4 t-3 b-2)-b^{3}\left(2-2 t+t^{2}\right) \\
& +2 b^{2} t\left(2-2 t+t^{2}\right)-a^{3}\left(2+3 b^{2}+b(6-10 t)-10 t+5 t^{2}\right) \\
& +a\left(2 b^{3}(t-1)+4(t-3) t^{2}-b^{2}\left(6-10 t+7 t^{2}\right)+4 b t\left(3-4 t+t^{2}\right)\right) \\
& \left.\left.\left.\left.\left.-a^{2}\left(b^{3}+b^{2}(6-8 t)-2 t\left(6-6 t+t^{2}\right)+b\left(6-18 t+11 t^{2}\right)\right]\right\}\right)\right)\right]\right\} \\
& +\varepsilon-\left\{\frac{B^{4} e^{-2(a+t)} a^{6}\left(2-2 e^{a+t}+(a+t)(2+(a+t))\right)^{2}}{2(a+t)^{6}}-\frac{A^{4} b^{2} e^{-2 t}}{2(t+b)^{2}}\right\}
\end{aligned}
$$

with $\varepsilon_{ \pm}$defined in Eq. (8). Here, the first $\left(\propto \varepsilon_{+}\right)$and second $\left(\propto \varepsilon_{-}\right)$terms are connected with the Coulomb interactions between the electrons and between them and their mirror 
images, respectively. The local field corrections are due to a many-body exchange effect in the 2DEG in the in-plane and given by [16]:

$$
G(t)=\frac{t}{2\left(t^{2}+t_{F}^{2}\right)^{1 / 2}}
$$

At very low temperature the phonon scattering is negligibly weak. Therefore the electrons are expected to experience the following scattering sources: i) ionized donors (ID), ii) alloy disorder (AD), iii) surface roughness (SR), iv) roughness-induced piezoelectric charges $(\mathrm{PE})$ and $\mathrm{v})$ roughness-induced deformation potential (DP). The total relaxation time is then determined by the ones for individual disorder according to Matthiessen's rule:

$$
\frac{1}{\tau_{\text {tot }}}=\frac{1}{\tau_{I D}}+\frac{1}{\tau_{A D}}+\frac{1}{\tau_{S R}}+\frac{1}{\tau_{P E}}+\frac{1}{\tau_{D P}} .
$$

\section{II.2. Autocorellation function for piezoelectric scattering}

In wurtzite III-nitride heterostructures, e.g. GaN/AlGaN, surface roughness gives rise to strain fluctuations in both strained and relaxed layers. In Ref. [17] Quang and coworkers have demonstrated that the strain fluctuations produce random nonuniform variations in the piezoelectric polarization. These in turn induce fluctuating densities of piezoelectric charges, viz. bulk charges of strained and relaxed layers as well as sheet charges on the interface. The charges create relevant electric fields and act as scattering sources on the $2 \mathrm{D}$ motion of electron in the in-plane. It has been pointed out [17] that the average electric field due to sheet charges is much weaker than those of bulk charges. In addition, the average field due to bulk charges in GaN well is nearly equal to that in the AlGaN barrier. Therefore, we may plausibly restrict ourselves to calculate the scattering by bulk charges located in the well layer. The potential energy for an electron moving in the field due to roughness-induced bulk piezoelectric charges in the channel layer is described by [12]:

$$
U_{P E}(\mathbf{q}, z)=\frac{\pi \alpha \epsilon_{\|} e Q}{\varepsilon_{L}} q \Delta_{q} F_{P E}(q, z) .
$$

Here, $\alpha$ denotes the anisotropy ratio as a measure for the deviation of hexagonal symmetry of the wurtzite crystal from isotropy, $\epsilon_{\|}$is the latice mismatch. Q is a material parameter characteristic of the well [17]. The form factor in Eq. (13) is given by

$$
F_{P E}(q, z)=\frac{1}{2 q}\left\{\begin{array}{lc}
e^{q z} 2 q L & \text { for } \quad z<0 \\
2 q z e^{q z}+2 e^{L q} \operatorname{Sinh}[q(L-z)] & 0<z<L \\
e^{-q z}\left(e^{2 L q}-1\right) & z>L
\end{array}\right.
$$

Upon averaging Eqs. (13) and (14) by mean of the lowest subband wave function from Eq. (1), we obtained the weighted potential for scattering by roughness-induced piezoelectric charges

$$
U_{P E}(\mathbf{q})=\frac{\pi \alpha \epsilon_{\|} e Q}{\varepsilon_{L}} F_{P E}(q / k) \Delta_{q}
$$


The weighted piezoelectric form factor is expressed as a function of the dimensionless wave number in Eq. (9):

$$
\begin{array}{r}
F_{P E}(t)=\frac{1}{\left.(a-t)^{4}\right)}\left[a ^ { 3 } L B ^ { 2 } \left(6-e^{t-a}(6+(6+(a-t)\right.\right. \\
(3+a-t))(a-t)))]+\frac{A^{2} b L e^{-t}\left(e^{2 t}-1\right)}{2 t(b+t)} \\
+\frac{1}{t\left(a^{2}-t^{2}\right)^{3}}\left[a ^ { 3 } B ^ { 2 } L e ^ { - a } \left(e^{a+2 t}(a-t)^{3}-e^{a} k^{3}(a+t)^{3}\right.\right. \\
\left.\left.+e^{t}\left(a^{2}(6+a(4+a)) t-2(a(2+a)-1) t^{3}+t^{6}\right)\right)\right]
\end{array}
$$

The scattering rate from piezoelectric charges are fixed by the interface profile and we have:

$$
\left\langle\left|\Delta_{\mathbf{q}}\right|^{2}\right\rangle=\pi \Delta^{2} \Lambda^{2} \frac{1}{\left(1+q^{2} \Lambda^{2} / 4 n\right)^{n+1}},
$$

where $\Delta$ is a roughness amplitude, $\Lambda$ is a correlation length and $\mathrm{n}$ is an exponent specifying the falloff of the distribution at large in-plane wave number in range $n=1$ to 4 .

\section{RESULTS AND CONCLUSIONS}

In this section, we are trying to apply the foregoing theory to understand transport properties of the 2DEG in wurtzite UID GaN/AlGAN SFQW. For numerical results, we have to specify parameters appearing in the theory as input. The lattice constant, elastic stiffness constants, piezoelectric constants, and dielectric constant for AlN and GaN are taken from Refs. [12,18]. The coresponding constants for an AlGaN alloy are estimated within the virtual crystal approximation [18]. The potential barrier heigh $V_{0}$, as usual, is to be equal to the conduction band offset between the $\mathrm{Al}_{x} \mathrm{Ga}_{1-x} \mathrm{~N}$ barrier and the $\mathrm{GaN}$ well, which depend on the $\mathrm{Al}$ content $x$ as $[19,20]$ :

$$
\Delta E_{c}=0.75\left[E_{g}(x)-E_{g}(0)\right],
$$

where the band gap of $A l_{x} G a_{1-x} N$ is given by [18]:

$$
E_{g}(x)=6.13 x+3.42(1-x)-x(1-x) e V .
$$

The anisotropy ratio $\alpha$ of the wurtzite $\mathrm{GaN}$ is chosen $\alpha=5[18]$ as a typical value in our numerical calculation.

We have carried out numerical calculations of the low-temperature 2DEG mobility due to piezoelectric scattering in UID GaN/ $\mathrm{Al}_{x} \mathrm{Ga}_{1-x} \mathrm{~N}$ SFQW . The effective electron masses of GaN are for the growth direction $m_{z}=0.18 m_{e}[15]$ and for the in plane $\mathrm{m}^{*}=$ $0.228 m_{e}[21]$. We are examining the key parameters to which the calculation of 2DEG mobility due to piezoelectric scattering is sensitive. We carried out calculation the 2DEG mobilities due to piezoelectric scattering versus electron density $n_{s}$, width of the well and thickness of strained GaN layer L, and an Al-content $x$. The numerical results are given in the figures 1 and 2. From calculation of results obtained, we may draw the following conclusions: 


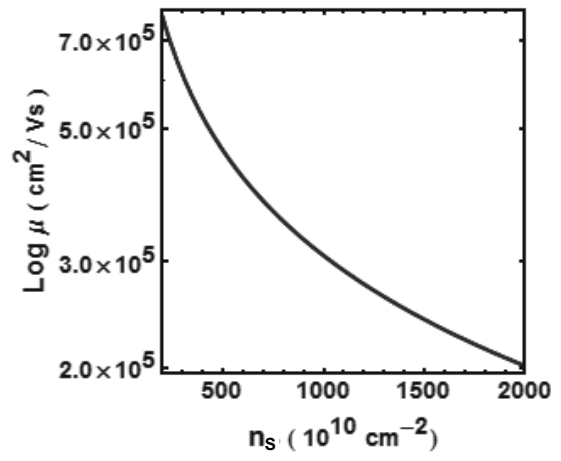

Fig. 1. 2DEG mobility of an UID $G a N / A l_{0.25} G a_{0.75} N$ SFQW vs. sheet electron density $n_{s}$.
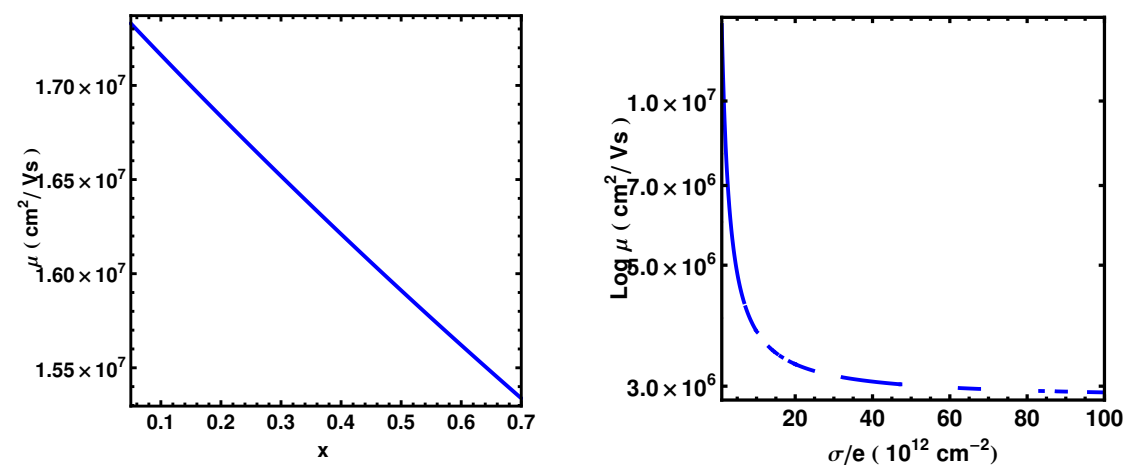

Fig. 2. 2DEG mobility of an UID GaN/AlGaN SFQW vs. an $\mathrm{Al}$ content $x$ and vs. density of sheet of polarization charge $\sigma / e$.

i) The electron mobility due to piezoelectric scattering is large. The piezoelectric scattering is one of the important scattering mechanisms in well [22].

ii) The electron mobility due to piezoelectric scattering decreases when density of sheet of polarization charge $\sigma / e$ increases because of polarization charges pulls 2DEG toward to barrier where are the key scattering sources. Due to numerical solving for minimum condition of Hamiltonial (2) is failed, some points on graph of the electron mobility were missing.

iii) Figure 2 reveals that owing the polarization charges induced from an abrupt change across the interface plane may strongly modify the functional dependence of a mobility on the $\mathrm{Al}$ content $x$ in addition to reducing it.

To summarize, in this contribution we have theoretically studied the electron mobility due to piezoelectric scattering and the effects of the polarization charges on mobility 
of the 2DEG in a UID GaN/AlGaN SFQW. We have derived analytical expression, which explicitly describes the scattering rates for piezoelectric scattering.

\section{ACKNOWLEDGMENT}

Financial support of Science and Technology Research program (project B2009-01268) is gratefully acknowledged.

\section{REFERENCES}

[1] D. Eger and Y. Goldstein, Phys. Rev. B19 (1979) 1089.

[2] Y. Grinshpan, M. Nitzan, and Y. Goldstein, Phys. Rev. B19 (1979) 1098.

[3] E. Veuhoff and D. Kohl, J. Phys. C14 (1981) 2395.

[4] G. Yaron, A. Many, and Y. Goldstein, J. Appl. Phys. 58 (1985) 3508.

[5] R. M. Cohen, M. Kitamura. and Z. M. Fang, Appl. Phys. Lett. 50 (1987) 1675.

[6] E. Yablonovitch, H. M. Cox, and T. J. Gmitter, Appl. Phys. Lett. 52 (1988) 1002.

[7] J. Dreybrodt, A. Forchel, and J. P. Reithmaier, Phys. Rev. B48 (1993) 14741.

[8] K. Kishimoto, Y. Shiraki, and S. Fucatsu, Thin Solid Films 81 (1998) 321; Appl. Phys. Lett. 70 (1997) 2837.

[9] J. F. Muth, X. Zhang, A. Cai, D. Fothergill, J. C. Roberts, P. Rajagopal, J. M. Cook, Jr. E. L. Piner, and K. J. Linthicum, Appl. Phys. Lett. 87 (2005) 192117.

[10] J. Lindhart, M. Scharff, and H. E. Schiott, Kong. Danske Vid. Selsk, Mat.-Fis. Medd. N.13 (1963) 33

[11] T. Ando, J. Phys. Soc. Jpn. 51 (1982) 3893; J. Phys. Soc. Jpn. 51 (1982) 3900.

[12] D. N. Quang, V. N. Tuoc, N. H. Tung, N. V. Minh, and P. N. Phong, Phys. Rev. B72 (2005) 245303.

[13] Nguyen Viet Minh, Physics and Engineering in Evolution, Publishing House for Science and Technics, (2008) 13.

[14] T. Ando, A. B. Fowler, and F. Stern, Rev. Mod. Phys. 54 (1982) 437.

[15] L. W. Wong, S. J. Cai, R. Li, K. Wang, H. W. Jang, and M. Chen, Appl. Phys. Lett. 73 (1998) 1391.

[16] M. Jonson, J. Phys. C9 (1976) 3055

[17] D. N. Quang, V. N. Tuoc, N. H. Tung, N. V. Minh, P. N. Phong, Phys. Rev. B72 (2005) 115337.

[18] O. Ambacher, B. Foutz, J. Smart, J. R. Shealy, N. G. Weimann, K. Chu, M. Murphy, A. J. Sierakowski, W. J. Shaff, I. F. Eastman, R. Dimitrov, A. Mitchell, and M. Stutzmann, Appl. Phys. 87 (2000) 334 .

[19] G. Martin, S. Strite, A. Botchkarev, A. Agarwal, A. Rockett, and H. Morkoc, Appl. Phys. Lett. 65 (1994) 610.

[20] G. Martin, A. Botchkarev, A. Rockett, and H. Morkoc, Appl. Phys. Lett. 68 (1996) 2541.

[21] L. Hsu and W. Walukiewicz, J. Appl. Phys. 89 (2001) 1783.

[22] Nguyen Viet Minh, unpublished (2009). 